Revue de l'Institut des langues et cultures

d'Europe, Amérique, Afrique, Asie et Australie

$34 \mid 2019$

Femmes et migrations auX $\mathrm{XIX}^{\mathrm{e}}$ et $\mathrm{XX} \mathrm{X}^{\mathrm{e}}$ siècles :

regards et représentations

\title{
Les « Boches du Nord» : ces femmes françaises exilées et leur image en France entre 1914 et 1918
}

The "Boches from the North": These Exiled French Women and Their Image in France between 1914 and 1918

\section{Nicolas Charles}

\section{(2) OpenEdition}

Journals

Édition électronique

URL : http://journals.openedition.org/ilcea/5764

DOI : 10.4000/ilcea.5764

ISSN : 2101-0609

Éditeur

UGA Éditions/Université Grenoble Alpes

Édition imprimée

ISBN : 978-2-37747-074-7

ISSN : 1639-6073

\section{Référence électronique}

Nicolas Charles, «Les «Boches du Nord » : ces femmes françaises exilées et leur image en France entre 1914 et $1918 »$, ILCEA [En ligne], 34 | 2019, mis en ligne le 15 janvier 2019, consulté le 05 mai 2019. URL : http://journals.openedition.org/ilcea/5764 ; DOI : 10.4000/ilcea.5764

Ce document a été généré automatiquement le 5 mai 2019.

(C) ILCEA 


\section{Les « Boches du Nord»: ces femmes françaises exilées et leur image en France entre 1914 et 1918}

The "Boches from the North": These Exiled French Women and Their Image in France between 1914 and 1918

Nicolas Charles

\section{Introduction}

Quelques jours après le début de la Première Guerre mondiale les Allemands envahissent le royaume de Belgique, pourtant neutre. En peu de temps, la petite armée belge qui se bat avec courage, est submergée, la majeure partie du pays occupée. Les troupes de Guillaume II foncent ensuite vers la frontière française, poussant devant elles un flot de réfugiés qui colportent de nombreuses atrocités ${ }^{1}$ commises par les armées du Kaiser. En arrivant en France, ces réfugiés créent un mouvement de panique. Ceux-ci sont essentiellement des personnes relativement aisées ${ }^{2}$ qui ont les moyens de fuir et surtout de la famille pouvant les accueillir loin du front. Dès le début des combats, les réfugiés et déplacés ${ }^{3}$ sont des victimes collatérales de l'invasion puis de l'occupation allemande. En effet, une fois le front stabilisé, à l'automne 1914, le flot de personnes en provenance des zones de combat ne se tarit pas ${ }^{4}$. À partir de 1915, les Allemands évacuent toutes les bouches inutiles afin de limiter les problèmes de ravitaillement ${ }^{5}$. Nous allons dans ce présent article, parler des femmes qui, durant toute la guerre ont dû, pour diverses raisons, quitter leur domicile dans le nord de la France. Des malheureuses du temps de l'invasion, leur image évolue peu à peu pour s'incarner dans celle des "Boches du Nord " au fur et à mesure que la guerre s'enlise : elles deviennent alors suspectes pour le reste des Français. Cette méfiance est d'ailleurs alimentée par l'État français qui interroge les civils en provenance des départements occupés afin d'obtenir des informations sur l'outre-front, mais aussi pour débusquer d'éventuels espions à la solde des puissances centrales qui tenteraient de pénétrer en France. Les femmes rapatriées ${ }^{6}$, en provenance 
des régions occupées, ont souvent parmi le reste de la population française une image déplorable : celle de la trahison car, dès les débuts de la guerre, de nombreuses rumeurs prêtent à ces dernières des relations intimes avec l'envahisseur, même si ce n'est pas uniquement le critère «sexuel» qui est à l'origine de la suspicion des Français de l'arrière. Dans de nombreux interrogatoires des autorités françaises à l'arrivée des rapatriés par la Suisse, le statut des femmes est systématiquement appréhendé : est-ce que ce sont des personnes coupables de trahison? Sont-elles «sûres » ou "suspectes »? L'image des «Boches du Nord» renvoie donc particulièrement à celles des femmes qui sont suspectées d'offrir leur corps à l'ennemi et par là même de trahir leur patrie. Mais il n'y a pas que cela: les femmes en provenance du Nord, lorsqu'elles arrivent dans une autre région, sont aussi stigmatisées à cause de leur accent, de leur façon de vivre. Les critères de leur rejet par les populations locales, qui ne comprennent pas ces femmes ${ }^{7}$ sont donc pluriels: la peur de la trahison, le rejet socio-économique, les incompréhensions culturelles. Nous essayerons donc à travers notre propos de voir, à partir de sources publiques et privées, comment les réfugiées sont perçues par les Français qui les accueillent à partir de 1914 en « France libre » en tentant de montrer ce qui tient de la rumeur sur les «Boches du Nord». Ce terme usuel et sanctionné par la justice, ne désigne pas que les femmes et ne stigmatise pas seulement l'acte de " collaboration horizontale ». On désigne ainsi des hommes, des enfants, généralement pour leur infliger l'insulte suprême. Ces injures traduisent les inimitiés entre communautés, à l'œuvre dès 1914, marginalement, puis progressivement jusqu'à atteindre un "front du refus » en 1917. Il désigne souvent des réfugiés n'ayant même jamais vu un Allemand puisqu'ayant fui l'invasion, comme les Picards en 1918.

\section{Quitter le nord de la France au moment de l'invasion allemande}

2 À partir du mois d'août 1914, tous ceux qui sont partis, de leur propre initiative, de leur région d'origine lors de l'invasion germanique sont qualifiés de réfugiés. La peur pour sa vie et celle des membres de sa famille est une raison essentielle dans le choix de nombreuses personnes de laisser leur maison et une partie de leurs biens à la merci des ennemis. Mais tout le monde n'a pas pu, pour des raisons matérielles, fuir. Ainsi, nous le verrons plus loin, les premiers réfugiés qui quittent les régions du Nord-Est menacées par l'arrivée des troupes de Guillaume II appartiennent la plupart du temps aux catégories sociales les plus élevées. Les réfugiés moins aisés ont fui dans un deuxième temps, lorsque l'armée allemande s'est faite plus pressante ${ }^{8}$.

3 Revenons quelques instants sur la peur de l'ennemi en nous posant la question de sa représentation chez les civils français. En août 1914, les armées franco-britanniques sont malmenées par les soldats allemands, représentés dans la presse alliée comme des barbares ou des animaux qui violent un territoire neutre (le royaume de Belgique), pillent ses habitations, assassinent, violentent et torturent ses habitants. Les femmes sont donc souvent les premières victimes des Allemands, en particulier en Belgique. De nombreux récits de massacres et de viols sont colportés par les premiers réfugiés, ce qui a souvent pour conséquence de créer une vague de panique et un afflux plus important de réfugiés par la suite, en particulier de femmes qui ne veulent pas vivre cette situation'. Cette image désastreuse des troupes allemandes s'explique de plusieurs manières. Il s'agit tout d'abord de l'ennemi, il convient donc de lui donner une image déplorable qui doit 
renforcer la haine de tous ceux qui s'opposent à eux. La propagande alliée des débuts du conflit insiste donc sur les champs lexicaux et visuels de la barbarie et de l'animalité. Ceci est d'autant plus d'actualité lors de l'invasion allemande que des rumeurs folles circulent sur des exactions germaniques vis-à-vis des civils: viols, meurtres, mais surtout des scènes de tortures sont colportées par les réfugiés, ce qui terrorise les populations. C'est le cas par exemple de la rumeur autour des enfants belges qui ont eu des mains coupées. John Horne et Alan Kramer, spécialistes des exactions lors de l'invasion allemande de 1914 ont bien étudié le processus de diffusion des rumeurs et ses conséquences sur les populations civiles ${ }^{10}$.

Des massacres de civils ont toutefois bien été perpétrés par les Allemands, de nombreux cartons d'archives leur sont consacrés, que ce soit aux Archives nationales ou au SHD ${ }^{11}$. Les réfugiés français à leur arrivée à l'arrière du front colportent de nombreuses rumeurs sur les massacres allemands qui sont systématiquement amplifiés par la propagande française. Les femmes, souvent choquées par le départ précipité de leur domicile, diffusent de nombreuses rumeurs sur un grand nombre de viols et surtout de mutilations dont leurs congénères et des enfants auraient été victimes de la part des Allemands. Plus tard, les rapatriés à leur arrivée en France sont systématiquement interrogés sur les massacres éventuels de 1914. Il s'agit pour les autorités françaises, tout au long du conflit, de constituer des dossiers à charge contre les Allemands qui pourront servir après-guerre pour d'éventuels procès ${ }^{12}$, mais aussi pour influer sur les négociations de paix au moment où elles seront engagées. Il convient ici de s'arrêter sur les exactions allemandes, car leur impact sur l'imaginaire collectif des civils français se perpétue tout au long de la guerre (et sera même mis en avant à la Libération pour renforcer le côté tyrannique des Allemands). L'exemple du village de Haybes-sur-Meuse est très significatif pour comprendre dans quel état d'esprit se trouvent civils français et soldats allemands après quelques semaines de conflit. Le village est situé au nord du département des Ardennes, sur la Meuse, à quelques kilomètres de la frontière belge. La commune a été totalement incendiée et une soixantaine d'habitants sont exécutés par les soldats allemands qui viennent de perdre un des leurs dans des combats aux alentours de la localité. Comme en Belgique, notamment à Dinant ${ }^{13}$, la population civile est tenue responsable du meurtre de soldats allemands : ces derniers agissent en représailles sur les civils accusés d'être des francs-tireurs qui ralentissent l'avancée des troupes germaniques. Bercés par les récits de leurs pères passés par là lors de la guerre de 1870-1871, les armées de Guillaume II se méfient énormément des civils qui pourraient agir contre eux, comme ils l'ont déjà fait lors du précédent conflit. C'est sans doute cela qui explique l'extrême brutalité avec laquelle ils agissent lors de l'invasion en Belgique et dans le nord de la France. Cela engendre donc de véritables scènes de panique dans les territoires français les plus septentrionaux qui voient déferler sur eux ces ennemis honnis depuis 1870 venant une nouvelle fois les asservir et les massacrer ${ }^{14}$. La répétition des invasions en à peine plus de quarante ans, conjuguée aux rumeurs sur les massacres réels ou supposés, explique le départ de nombreux civils en août-septembre 1914. 


\section{Qui sont ces personnes qui affluent des départements frontaliers avec l'Allemagne ou la Belgique?}

Dans un premier temps, il s'agit de réfugiés. Les premiers à partir sont des personnes aisées qui ont les moyens de financer leur départ puis leur installation dans la localité de repli : à ce titre, beaucoup de familles bourgeoises font partie de la première vague de réfugiés. Dès les premières nouvelles de l'invasion allemande en Belgique, elles partent avec des moyens de transport plus rapides (trains, voitures). Puis, un flot de réfugiés plus pauvres succède à cette première vague, composant ce « chapelet de misère " hétéroclite décrit par de nombreux combattants. Ceux qui partent sont en général plus jeunes : il s'agit surtout de jeunes femmes ou de jeunes mères. Les personnes âgées le plus souvent décident de rester sur place. Autotal, seulement $20 \%$ de la population de ces dix départements occupés a fui.

Une fois arrivés loin de la zone des combats, les réfugiés doivent posséder une certaine fortune personnelle ou compter sur les solidarités familiales ou amicales dans leur localité d'accueil, car dès les débuts de la guerre, l'État français légifère sur les aides à fournir aux personnes qui ont quitté leur domicile à cause du conflit.

L'instruction ministérielle du 12 décembre 1914 sur « le rapatriement des réfugiés et évacués » disposant dans son paragraphe 3 que les familles françaises évacuées de leur résidence par l'autorité militaire ${ }^{15}$ seront rapatriées aux frais du département de la guerre [...].

J'ai l'honneur de vous faire connaître que c'est aux familles intéressées, qui sollicitent le bénéfice de ces décisions, qu'il appartient de faire la preuve (par production d'ordres écrits ou de témoignages officiels), qu'elles ont bien été évacuées de leur résidence par ordre.

Si la preuve ci-dessus n'est pas faite par elles, les familles devront être considérées comme ayant abandonné leur domicile de leur propre initiative et dans ce cas, subvenir elles-mêmes aux dépenses de leur rapatriement ${ }^{16}$.

7 Par la suite, à partir de 1915, les personnes en provenance des territoires français occupés par l'armée allemande sont qualifiées de rapatriés par les autorités françaises, car au départ, en 1915, ils ont été obligés par les militaires allemands de quitter leur domicile. Pour ce qui est des besoins du quotidien, les réfugiés et rapatriés doivent faire appel à la solidarité. Le plus souvent, ce sont des gens originaires de la même région se trouvant dans la localité d'accueil qui aident les émigrés ou qui mettent en place des réseaux d'entraide.

Qui sont ces personnes évacuées par les Allemands durant le conflit et pour quelles raisons?

Dès que les premiers effets du blocus maritime des alliés se font sentir en 1915, les Allemands se décident à évacuer les bouches inutiles des territoires qu'ils occupent afin de réserver le maximum de vivres pour leurs troupes. Comme la majorité des personnes restant dans les territoires occupés sont des femmes, celles-ci sont donc logiquement majoritaires à être évacuées par l'armée allemande. En effet, les rares hommes présents sont plus difficilement évacués puisqu'ils représentent à la fois une force de travail supérieure sur place et un danger potentiel de rejoindre les armées françaises en cas d'évacuation. Le trajet suivi est similaire dans de nombreux cas, comme nous le présente 
$\mathrm{M}^{\text {lle }}$ Chaptal : les personnes quittent les territoires occupés à partir d'une ville importante où elles sont regroupées dans des trains spéciaux à destination de la Suisse, pays neutre, via l'Allemagne. Le premier convoi de rapatriés arrive dans le pays neutre le 16 mars 1915 (Chaptal de Chanteloup, 1919: 15). Une fois entrés dans la Confédération helvétique par Schaffhouse, ils passent ensuite par Zurich, Berne, Lausanne puis Genève ${ }^{17}$. De là, ils sont dirigés vers la France via deux points d'entrée principaux : Évian et Annemasse. C'est là, nous le verrons plus loin, que l'État français met en place des commissariats spéciaux chargés d'interroger les évacués dans un but double : éviter la pénétration du territoire national par des espions germaniques et collecter des renseignements sur les territoires tombés sous la coupe de l'ennemi. Pour les civils envoyés en France, leur évacuation est due au manque de nourriture que les Allemands attribuent à « la France et l'Angleterre qui ont cherché, au mépris des accords internationaux et de la convention de La Haye, à provoquer la disette parmi les populations allemandes et en retour l'Allemagne se voit dans l'obligation de procéder à l'évacuation d'une partie des territoires occupés ${ }^{18}$ ». Nous le voyons donc, les occupants se dédouanent sur les Alliés pour justifier des évacuations qu'ils se disent obligés d'effectuer faute de nourriture suffisante pour tous. Des tracts en ce sens sont distribués aux évacués en transit en Allemagne en avril 1915 où il est écrit que ces évacuations sont de la responsabilité du gouvernement français qui a refusé de « fournir, par la voie des pays neutres, les aliments nécessaires aux habitants des localités situées dans la région occupée par les troupes allemandes ${ }^{19} »$. La propagande allemande donne donc l'image d'un gouvernement français ayant abandonné les départements situés de l'autre côté du front. De leur côté, les autorités françaises sont méfiantes vis-àvis de cet afflux d'évacués. Pour le ministère de l'Intérieur, il s'agit pour les Allemands de "vider» les territoires occupés pour que les armées du Kaiser réquisitionnent plus facilement nourriture, matériels ou logements à destination de leurs troupes. En mars 1915, dans son rapport hebdomadaire au ministre de l'Intérieur, le commissaire spécial envoyé à Annemasse pour interroger les évacués donne un autre point de vue :

L'évacuation systématique imposée est un moyen qui a paru bon pour peser sur l'opinion publique en France. On paraît escompter que l'arrivée constante de gens venant d'un pays ruiné en partie apportera une gêne parmi les populations appelées à les hospitaliser, dont le développement hâterait la conclusion d'une pays honorable ${ }^{20}$.

Les évacués se retrouvent donc au cœur de la propagande des deux camps qui se renvoient la responsabilité de ce déboire humanitaire.

\section{Pourquoi partir en plein conflit ?}

Les motivations des civils français pour quitter les territoires occupés sont diverses. Il convient ici de montrer que ce mouvement d'évacuation n'est en aucun cas linéaire. Dans les premiers temps, les habitants des régions occupées semblent préférer rester sur place. Il s'agit pour eux de préserver au maximum leurs biens des différents pillages organisés par l'administration germanique. C'est la même raison qui explique que ces personnes n'ont pas fui lors de l'invasion de 1914. Ainsi à Tourcoing, dans le département du Nord, dans le premier convoi de mars 1915 à destination de la France, il n'y a pas assez de volontaires : les Allemands ont complété le train avec un tirage au sort parmi les familles les plus pauvres de la ville ${ }^{21}$. À Sedan dans les Ardennes, les autorités allemandes affichent l'ordre suivant : « Les personnes qui ne se conforment pas aux prescriptions [...] sont prévenues qu'elles y seront contraintes par la force, et, en outre, à titre de 
représailles, il leur sera retiré l'autorisation d'emporter des bagages.» (Stephani, 1919:92) Dans ce même département des Ardennes, des témoignages de diaristes montrent qu'en 1915, des personnes choisies pour être évacuées se cachent des Allemands où ils peuvent: dans les champs ou les forêts, si bien que les autorités militaires sont obligées de mener des arrestations massives pour remplir les convois d'évacuation prévus ${ }^{22}$. Mais, au fil du temps, face à une occupation qui devient de plus en plus difficile, les refus d'évacuer sont beaucoup moins nombreux : ainsi, sur 750 Lillois qui arrivent à Annemasse le 15 décembre 1915, cinq seulement ont été contraints de quitter les territoires occupés ${ }^{23}$. À partir de là, une inversion de tendance est nettement visible : beaucoup de civils, surtout des femmes, cherchent par tous les moyens à quitter les territoires administrés par les Allemands. Ainsi Eugénie Deruelle, de Sains-Richaumont dans l'Aisne, qui se montrait hostile aux évacuations dans son carnet (Deruelle, 2010) ${ }^{24} \mathrm{a}$ changé complètement d'avis en 1917 : «Le départ de mes parents m'a causé une vive émotion; je crois qu'ils ont bien raison de quitter notre pauvre pays qui sera le théâtre d'événements terribles et où nous courrons le risque de mourir de faim.» (Ibid.: 78 [28 juin 1917]) À partir de 1916, tous les moyens sont bons pour faire partie des convois destinés à être envoyés vers la «zone libre». Ainsi, à Saint-Quentin, dans l'Aisne, plusieurs personnes, à leur arrivée à Évian ou Annemasse, témoignent avoir versé de fortes sommes d'argent (un ou deux louis d'or) ou fait des dons en nature à des officiers allemands pour que leur nom soit inscrit sur les listes d'évacuation ${ }^{25}$. Parfois, les personnes désireuses d'être envoyées en zone non occupée passent par l'intermédiaire de collaborateurs avérés pour obtenir le droit d'être évacuées. C'est le cas à Roubaix dans le Nord où une fleuriste de la ville, maîtresse d'un officier allemand, reçoit de la part de ses concitoyens de nombreux cadeaux pour faciliter leurs démarches en vue de l'évacuation ${ }^{26}$ . Dans la commune voisine de Mons-en-Barœul, une jeune femme avoue à son arrivée en France avoir pu quitter le Nord grâce aux démarches de sa sœur qui était employée à la Kommandantur locale ${ }^{27}$. Ces cas de pots-de-vin ou de compromission avec l'ennemi pour obtenir le précieux sésame vers la France étaient nombreux : cela fait partie des rites d'accommodement régulièrement pratiqués par de nombreux civils pour améliorer leur sort. Ce genre de compromission va être une des raisons, mais pas la seule, à la naissance dans le reste de la population française du mythe des «Boches du Nord». Toujours est-il que les Allemands ne sont pas dupes et durcissent, à partir de 1917, les conditions à remplir pour être évacué, ce que confirment deux jeunes femmes originaires de Lille :

Pour obtenir d'être évacuées, les Allemands exigeaient de nous des démarches pénibles et multiples; ils semblaient consentir et toujours il fallait recommencer et être reçues comme des chiens. Ils ne voulaient laisser partir aucune jeune fille qui pût travailler pour les soldats en France. Un jour nous apprîmes que nous étions inscrites pour la déportation ${ }^{28}$ et puis subitement ils changèrent d'avis et nous firent recommencer toute la démarche en vue de l'évacuation. (D'Alix, 1919)

11 Comme le montre cet extrait, pour les hommes et les femmes âgées de 14 à 45 ans, il est de plus en plus difficile de faire partie des convois à destination de la Suisse. En effet, l'administration germanique a peur de laisser partir des personnes qui pourraient renforcer le potentiel militaro-industriel français en travaillant dans les usines d'armement. En 1918, avec l'apparition de l'épidémie de grippe espagnole, les convois diminuent pour éviter les grands rassemblements de personnes propices à la circulation de la maladie : après l'été 1918, les autorités françaises ferment leurs frontières avec la Suisse dans ce cadre-là. Cette mesure radicale s'explique aussi par les succès des offensives alliées de l'été 1918 qui libèrent de nombreux territoires: plus besoin 
d'accueillir des rapatriés puisque les régions occupées par les Allemands depuis 1914 sont peu à peu reconquises. Les commissariats spéciaux d'Évian et d'Annemasse destinés à accueillir et interroger les civils rapatriés cessent leurs activités en janvier 1919.

Quels chiffres pour les Français rapatriés entre 1914 et 1918? Au vu de différentes sources, il semble que l'on puisse estimer à 500000 personnes (Nivet, 2011 : 311) évacuées par les Allemands entre 1914 et 1918. Le nombre d'évacués varie bien sûr selon les différents départements. Dans les Ardennes, seul département entièrement occupé, 70000 personnes (ibid.) sont évacuées entre 1915 et 1918. Il s'agit ici d'un département majoritairement rural où la population est moins élevée que dans le Nord. Ainsi, à Lille, plus de 30000 personnes dont plus de 20000 femmes (ibid.) font partie des convois envoyés vers la France via la Suisse sur la durée du conflit. Celle-ci, du fait de la mobilisation en 1914, du départ de nombreux réfugiés en 1914 puis des évacuations entre 1915 et 1918, a perdu la moitié de sa population entre 1913 et $1918^{29}$. Cette baisse démographique est très visible dans de nombreuses villes des Ardennes: Rethel, proche du front, n'a plus que 1600 habitants en novembre $1917^{30}$; Mézières la préfecture du département 4000 et Charleville, la plus grande ville des Ardennes, est passée à la fin de 1917 en-dessous de 9000 habitants ${ }^{31}$. En 1918, les territoires occupés ont donc perdu, entre la mobilisation des hommes en 1914 puis les différentes vagues de départs tout au long de la guerre, la majorité de leur population d'avant-guerre. Nous le voyons donc, entre les départs volontaires au moment de l'invasion puis les évacuations pratiquées par les Allemands durant la suite du conflit, plus de deux millions de civils français ont quitté leur domicile. Il s'agit d'un mouvement de population majeur dans l'histoire de la France au $\mathrm{Xx}^{\mathrm{e}}$ siècle, après bien sûr celui de l'exode ${ }^{32}$ massif de mai-juin 1940. Face à ces grands déplacements de population, les Français s'organisent dès le mois d'août 1914 pour accueillir toutes les personnes qui ont fui les Allemands. À partir de ces chiffres, combien de femmes ont quitté leur région d'origine durant ce conflit? Un chiffre précis est difficile à avancer: nous pouvons simplement dire que la majorité des personnes évacuées par les Allemands sont de sexe féminin, nous avons vu plus haut pourquoi. C'est la même chose pour les réfugiés qui ont fui les premiers combats: sur plus de deux millions de personnes déplacées depuis les régions occupées, nous pouvons affirmer que ce sont majoritairement des femmes. À partir des archives des départements de l'arrière nous pouvons dresser des statistiques. Dans l'Ouest (voir Richard, 2004), les femmes représentent $37 \%$, les enfants $44 \%$ et les hommes $18 \%$. Si l'on inclut donc les filles mineures, les femmes sont majoritaires.

\section{L'État français organise des interrogatoires à l'arrivée des rapatriés}

13 Les commissariats spéciaux d'Évian et d'Annemasse sont mis en place pour canaliser l'arrivée de civils évacués par les Allemands. Ils se trouvent dans ces deux communes de Haute-Savoie, car elles sont frontalières de la Suisse, et donc points d'entrée privilégiés sur le territoire français puisque l'administration allemande a choisi l'évacuation via la Confédération helvétique, pays neutre. Il n'y a donc pas d'échanges directs entre les belligérants, qui se servent de la Suisse comme lieu de transit. L'ouverture de ces commissariats spéciaux doit répondre à une double problématique : éviter la pénétration de l'espace français par des espions à la solde des Empires centraux et recueillir un maximum d'information sur la situation dans les territoires occupés afin de préparer leur 
libération le moment venu. C'est ainsi que, dès leur arrivée en France, les évacués subissent des interrogatoires longs et précis de la part d'officiers militaires mais aussi d'agents du ministère de l'Intérieur. Cette cogestion des commissariats spéciaux s'explique par le double but qui leur est assigné à leur création : le ministère de l'Intérieur lutte contre l'espionnage et prépare la fin du conflit, c'est-à-dire le moment où il faudra juger les personnes coupables de collaboration; le ministère de la Guerre cherche, lui, à récolter des informations précises sur les positions allemandes et leur organisation au sein des territoires perdus afin de préparer au mieux les offensives en direction de ces secteurs. Ces interrogatoires sont normés: ils contiennent plusieurs rubriques qui doivent permettre aux fonctionnaires à la fois d'obtenir des renseignements sur la personne interrogée et sur sa localité d'origine. Comme le montrent les documents concernant l'interrogatoire de Théophile Gacoin, originaire d'Avaux-le-Château dans les Ardennes le 11 décembre 1916 (Service historique de la Défense, 16 N 1226), les informations recueillies sont très denses. Chaque fiche commence par des renseignements personnels. Ainsi, l'âge, le domicile et l'emploi de la personne interrogée apparaissent. Cette fiche permet aussi de connaitre le temps de trajet entre les territoires occupés et l'arrivée en France libre. Dans le cas présent, ce réfugié a mis deux jours pour atteindre Annemasse depuis les Ardennes, ce qui est relativement rapide, compte tenu des nombreux arrêts du convoi en traversant les territoires occupés puis l'Allemagne et enfin la Suisse ${ }^{33}$. Ensuite, la fiche s'intéresse aux Allemands dans la commune : types de troupes présentes dans la localité, leur armement, la localisation de leur quartier général, les travaux de défense effectués pour renforcer leur position, état du ravitaillement des troupes ainsi que leur moral. Une fois les renseignements d'ordre militaire traités, la suite de l'interrogatoire concerne directement la vie quotidienne dans la commune d'Avaux-leChâteau. Il s'agit pour les autorités françaises de s'informer sur l'état sanitaire des populations civiles occupées. Les fonctionnaires cherchent ensuite à connaître le fonctionnement administratif de la commune. Dans le cas étudié ici d'Avaux-le-Château, Théophile Gacoin nous apprend que le maire est toujours en poste, mais que ce sont les Allemands qui administrent la commune: mise en place de papiers d'identité et de laissez-passer pour se déplacer dans la commune, interdiction de s'éloigner de celle-ci, appel mensuel des habitants pour éviter que certains cachent des clandestins venus espionner pour le compte des Alliés. Ces mesures décrites ici se retrouvent dans la plupart des procès-verbaux d'interrogatoires des rapatriés passés par Évian ou Annemasse. Ils montrent tous la rigueur de l'occupation militaire allemande qui laisse peu de libertés aux civils. Nous pouvons ici noter que dans la majorité des procès-verbaux que nous avons dépouillés dans les différentes archives, il y a peu de questions sur les travaux forcés infligés par les occupants aux civils. Il ne s'agit pas d'indifférence de la part des fonctionnaires quant au sort de leurs compatriotes, mais sans doute plutôt de manque d'intérêt stratégique : pour les autorités françaises il s'agit de connaître l'état des territoires perdus pour faciliter leur reconquête plutôt que de savoir le sort que réservent les autorités germaniques aux civils; c'est de la Realpolitik. D'ailleurs, il est frappant de remarquer que les mentions sur les réquisitions et les pillages par les Allemands sont nombreuses : encore une fois, c'est l'état économique des régions occupées qui préoccupe les autorités françaises. L'intérêt des autorités françaises pour l'attitude des civils, surtout des femmes, pendant l'occupation est prégnant. La forme peut varier, mais les rapatriés doivent toujours noter les personnes « sûres $^{34}$ » et les personnes « suspectes ${ }^{35}$ » dans la commune. Le procès-verbal de l'interrogatoire que nous étudions ici montre essentiellement les personnes suspectes: un collaborateur («il s'affiche avec les 
Allemands et les reçoit régulièrement chez lui $\left.{ }^{36} »\right)$, des femmes qui pratiquent la collaboration horizontale ( beaucoup de femmes font la noce. Cinq ont eu des enfants avec des soldats ennemis»). Ce type de dénonciations de personnes suspectes de collaboration est présent dans tous les interrogatoires. Cela ne veut pas dire que la collaboration se pratiquait à grande échelle dans les territoires occupés. Simplement, cela traduit les difficultés à juger un comportement : une personne qui va régulièrement à la Kommandantur ne collabore pas forcément. Il faut donc ensuite, pour le chercheur qui travaille sur les comportements sociaux en période d'occupation, croiser les sources avec d'autres témoignages et surtout avec les procès qui se sont tenus après la guerre. Cela permet de voir que la majorité des cas dénoncés dans les interrogatoires de rapatriés ne sont pas des cas de collaboration avérés. C'est la même chose pour les cas de collaboration horizontale : il s'agit de rester prudent vis-à-vis des femmes accusées d'avoir des relations sexuelles avec les soldats allemands. Sont-elles consenties ou subies? Les cas de viols sont nombreux lors de l'invasion (Horne \& Kramer, 1994) et ne sont que rarement dénoncés comme tels par les femmes qui ont peur des représailles allemandes et de l'opprobre de leurs concitoyens. De même, il faut comprendre les raisons qui poussent certaines femmes à se donner à l'ennemi : beaucoup d'entre elles le font pour pouvoir survivre. Dans tous les cas, les personnes évacuées qui dénoncent leurs compatriotes ne cherchent pas à comprendre ou à expliquer les comportements de leurs concitoyens. Elles répondent simplement à des questions sans vérifier leurs informations, parfois même en colportant des réputations qui ne sont pas avérées. Des réfugiés vont même jusqu'à inventer des faits de collaboration pour se venger de certains individus.

Les autorités françaises se servent donc des rapatriés comme d'une source de premier ordre pour recueillir des informations sur les territoires occupés. S'engage ensuite pour les fonctionnaires des commissariats spéciaux un immense travail de collecte des informations et de recoupement des témoignages qui a pour but de dresser un état des lieux précis des zones occupées. Il s'agit de dresser des listes de personnes sur lesquelles l'État pourra s'appuyer lors de la libération. Ces mêmes listes révèlent le nom des personnes suspectes de collaboration permettant l'évacuation de ces dernières le moment venu pour éviter des vengeances sauvages et d'assurer le jugement dans le respect des lois de la République. Ainsi en 1918, lorsque les territoires sont libérés, l'autorité républicaine est tout de suite rétablie : il n'y a pas de vacance du pouvoir propice aux vengeances populaires en France ${ }^{37}$ lors de la Grande Guerre ${ }^{38}$.

\section{L'image des rapatriés auprès du reste de la population française : "les Boches du Nord » ${ }^{39}$}

Une fois autorisés à entrer sur le territoire français, les rapatriés ont deux choix : aller séjourner chez une connaissance ou un membre de leur famille en laissant une adresse précise pour que les autorités françaises puissent les contacter, ou aller dans une commune choisie par l'État pour y être accueillis durant le reste du conflit. La plupart des réfugiées, ne sachant pas où aller, sont prises en charge par l'administration française qui les envoie sur tout le territoire, dans des secteurs plutôt éloignés du front ${ }^{40}$. Ainsi, la partie méridionale du pays reçoit les plus gros contingents, ce qui, nous le verrons plus loin, va poser de nombreux problèmes quant à leur intégration. Une fois arrivées dans les communes d'accueil, elles sont reçues par le maire qui les loge dans des bâtiments inoccupés ou parfois appartenant à la commune. Jusqu'en 1918, le cantonnement collectif 
est prohibé ; les rapatriées sont donc logées en général chez les habitants. Puis, vers la fin du conflit, par nécessité, des logements collectifs sont créés afin de pouvoir loger tout le monde. Comme beaucoup ont moins de treize ans ${ }^{41}$, elles se rendent à l'école du village où l'instituteur les accueille et travaille avec elles sur leur vie passée dans les territoires occupés par les Allemands. Il répond en cela à une demande du ministère de l'Instruction publique qui souhaite obtenir, lui aussi, des informations sur les territoires perdus. De nombreux travaux d'instituteurs sont disponibles aux Archives nationales ou à la BDIC sur ce sujet. Afin d'étayer notre propos, nous nous intéresserons ici au sort d'enfants meusiens évacués et logés dans les Alpes-Maritimes. Parmi les différents travaux proposés aux jeunes émigrés par leurs enseignants, le plus récurrent est «vous raconterez cette scène de vandalisme et essayerez de montrer tout ce que la rage de détruire révèle chez les Allemands de bassesse et de légèreté » (Archives départementales de la Meuse, $10 \mathrm{R}$ 193). Ce type de sujet induit indubitablement de nombreux récits sur l'invasion allemande et les exactions concomitantes. Ainsi, l'esprit des enfants qui écrivent sur ce thème est encore, en $1916^{42}$, tout imprégné de la violence de ces événements qui ont bouleversé leur jeune vie.

Suzanne, de Béthincourt, montre dans sa copie la violence de l'ennemi : les menaces de mort sont récurrentes lors de l'invasion, à tel point que le village doit être évacué — « plusieurs officiers allemands qui, revolver au poing, menaçaient tout le monde, les femmes, les enfants et les vieillards» (Archives départementales de la Meuse, $10 \mathrm{R}$ 193). Ce témoignage est représentatif de l'ensemble du corpus contenu dans ce dossier des Archives départementales de la Meuse. L'invasion est vraiment le moment marquant que les enfants ont choisi de relater pour identifier et caractériser les violences germaniques. En recueillant ces témoignages, les enseignants s'inscrivent dans une démarche de propagande globale en place dans tous les pays belligérants: il s'agit de montrer l'inhumanité de l'ennemi et de le stigmatiser pour rendre la guerre qui lui est faite juste. Dans un conflit en voie de totalisation, les enfants, surtout les filles, symboles d'innocence et de pureté, sont donc aussi mis à contribution par les autorités pour trouver des arguments contre les Allemands. Mais cette promiscuité subie lors de l'occupation va aussi créer chez de nombreux Français l'idée que les rapatriés peuvent être de mauvais Français. Une expression est alors formulée pour les qualifier : « les Boches du Nord ».

17 Une fois arrivés dans leur lieu de repli, les rapatriés sont donc installés dans des logements vacants, parfois dans des hôtels. Marcel Gaucher, habitant de Grasse dans les Alpes-Maritimes, montre qu'ils sont nombreux à arriver dans certaines communes : « Les restrictions alimentaires nous étaient épargnées. Cette situation privilégiée nous valut d'accueillir des trains entiers de réfugiés qui venaient grossir la population grassoise.» (Nivet, 2004 : 105) Les enfants, nous l'avons vu, vont à l'école : c'est le premier lieu où ils subissent railleries et vexations quant à leurs origines. Cela est exacerbé dans le Midi où les langues régionales, très usitées dans les campagnes, sont une barrière à l'intégration. Même si le français est parlé et compris partout dans l'hexagone, la langue régionale est un facteur d'identification fort au début $\mathrm{du} \mathrm{xx}^{\mathrm{e}}$ siècle. Ainsi, dans le sud-ouest du pays, les différents dialectes occitans sont la langue maternelle avant le français. À l'école, les enfants de rapatriés sont mis à l'écart par les autres enfants originaires du cru, car la langue qu'ils utilisent est elle aussi un patois local du Nord, incompréhensible pour les Méridionaux. Pour ces derniers, les dialectes proches du flamand s'apparentent à des langues germaniques : le mythe des «Boches du Nord» est donc avant tout linguistique. Cette barrière de la langue renforce l'incompréhension des populations qui accueillent les 
rapatriés : parfois à l'écart dans la commune d'accueil, ils ont tendance à rester entre eux. Comme tout le monde juge cette situation temporaire il n'y a pas de velléité d'intégration de part et d'autre. L'expression «Boches du Nord» provient aussi de la situation d'occupation que subissent les régions frontalières du Nord et de l'Est. Pour les Français habitant loin du front, vivre avec l'ennemi est incompréhensible, d'autant plus lorsque les hommes de la famille sont partis si loin pour défendre leur pays et venir en aide à ces compatriotes occupés. De nombreuses familles ont l'impression de payer le prix du sang pour une guerre lointaine qui ne les concerne pas directement. L'Allemand, le "Boche", n'est connu que par la presse et les représentations faites par les caricaturistes qui les assimilent à des monstres. Ceux qui accueillent les réfugiés ne comprennent pas comment on peut vivre et cohabiter avec les Allemands, à moins de collaborer. Un jeune émigré meusien, Eugène, raconte qu'à Spéracédès dans les Alpes-Maritimes, « il y a des gens bons et serviables mais beaucoup ne sont pas humains, ils nous traitent d'Allemands et de Boches. Ils ne savent pas ce que c'est la guerre et la misère » (Archives départementales de la Meuse, $10 \mathrm{R}$ 193). Pour la majorité des Français, l'expression «Boches du Nord", employée pour qualifier les rapatriés, est synonyme de traîtrise et de collusion avec l'ennemi. Ils ne voient dans les rapatriés que des personnes qu'ils pensent assistées par l'État, bénéficiant donc de nombreuses prestations sociales pour les aider à vivre. C'est en grande partie de cette incompréhension de la situation humanitaire dramatique des réfugiés que l'imaginaire collectif français dans les années d'après-guerre garde en mémoire cette expression infamante de «Boches du Nord ». Cette mémoire renvoie donc en particulier aux femmes : leur mari au front, la présence d'Allemands, un dialecte qui peut paraître proche de celui de l'ennemi, tout porte à croire chez les civils qui les accueillent que ces femmes ont pu trahir leur pays. Nous l'avons vu plus haut, des cas ont existé. Mais il convient de noter que la majorité de celles qui ont pratiqué la collaboration horizontale est restée sur place, avec leur amant, dans les territoires occupés.

Certaines les ont même suivis en Allemagne après la fin du conflit, comme Louise Guérin, habitante de Mézières (Ardennes) (Lambert, 1995: 29-42). À Lille, en 1919, plus de 50 procès pour divorce sont intentés par des soldats français qui, en rentrant chez eux, se sont aperçus que leur femme avait quitté la région avec leur amant germanique. Il existe également un camp d'internées civiles pour "femmes douteuses» dans le sud du Finistère (Richard, 2002) qui abrite une cinquantaine de femmes soupçonnées d'avoir entretenu une liaison avec l'Allemand. Ce faible nombre démontre toutefois que cette préoccupation, effective dans les interrogatoires, ne se traduit pas par une flambée des accusations objectives. Ce grief est marginal dans l'utilisation du terme «Boche du Nord » par rapport à la barrière linguistique ou à d'autres critères socio-culturels énoncés plus haut.

\section{Conclusion}

Entre 1914 et 1918, plus de deux millions de personnes ont fui les territoires passés sous le contrôle des troupes de Guillaume II dans le nord et l'est de la France. Que ce soit des réfugiés qui, en 1914, sont partis à cause de l'invasion allemande, ou des évacués qui ont pu quitter les zones administrées par l'ennemi à partir de 1915, elles ont en commun d'avoir été déracinées par la guerre et poussées, durant la durée du conflit tout du moins, sur les routes de l'exil. Ce chiffre de 2 millions de « déracinés » est une estimation : nous avons vu que les rapatriés étaient environ 500000 ; il y a donc eu sans doute plus de 
1,5 million de réfugiés entre 1914 et $1918^{43}$. À travers cet article, nous avons essayé de montrer que ces femmes réfugiées ont été des victimes à part entière de ce conflit. Pourtant, l'historiographie française de la Première Guerre mondiale les a longtemps laissées de côté : elles ont été, pour paraphraser Annette Becker (2012), dont les travaux sont pionniers sur le sujet, les « oubliées de la Grande Guerre » puisque la majorité des personnes déplacées durant ce conflit sur le front ouest étaient des femmes, comme nous avons pu le voir plus haut avec des statistiques dressées à partir des départements d'arrivée. Les autorités françaises ont ainsi pu recueillir de nombreuses informations à destination des différents ministères qui géraient le conflit et ses conséquences. Ces procès-verbaux, nous l'avons vu, sont aujourd'hui une source primordiale pour qui s'intéresse à la vie quotidienne dans les territoires occupés. Mais c'est le qualificatif de «Boches du Nord » employé pour nommer ces personnes par les autres civils français qui reste un fait marquant de ce conflit. En effet, nous sommes ici loin de l'unité nationale voulue, dès 1914, par les autorités dans le cadre de l'Union sacrée. Loin des images de propagande qui montrent un peuple français uni derrière ses soldats, la question des «Boches du Nord" met en lumière un aspect longtemps méconnu de ce conflit. C'est peut-être aussi ce qui explique que l'étude des réfugiés et de la vie quotidienne dans les territoires occupés ait longtemps été le parent pauvre de l'historiographie de la Grande Guerre. Si de nombreuses études sur l'occupation allemande sortent dans les années 1920, elles sont à charge car elles traitent généralement des exactions allemandes et ont un écho uniquement local. Les Français des autres régions, touchés par la perte d'un des leurs au combat, ne prêtent aucune attention au sort des habitants des anciennes régions occupées, encore moins à ceux qui ont dû les fuir. De plus, dans une société encore largement dominée par les hommes, le sort des femmes importe ici très peu: il vaut mieux rendre hommage au sacrifice des Poilus plutôt qu'à celui des femmes qui ont eu à subir les affres de l'occupation. Preuve de l'ambiance «machiste » de l'époque, la France ne donne pas le droit de vote aux femmes à la fin du conflit, contrairement à l'Allemagne ou au Royaume-Uni. Les réfugiées et les occupées sont donc bien des oubliées de cette Grande Guerre. La plupart de celles qui ont quitté ces régions meurtries rentrent dès la fin des combats, au plus tard dans le courant de l'année 1919. Elles trouvent des communes détruites par les combats et les pillages de l'armée allemande. Les quatre années de guerre ont laissé une marque indélébile dans les paysages, mais aussi dans les esprits. D'ailleurs, dans plusieurs journaux intimes écrits durant le conflit par des femmes qui ont dû fuir leur région occupée, l'amertume domine : celle d'avoir laissé les siens et ses biens aux mains de l'ennemi mais, plus encore, celle d'être mal reçue par des compatriotes qui ne comprennent pas leur situation. Enfin, celle de ne pas être reconnues, après-guerre, comme des victimes car, si des récompenses pour les civils sont créées par l'État ${ }^{44}$, peu ont été remises à des réfugiées. Preuve que leur sort, aussi bien pendant qu'après le conflit, a peu ému la population française. 


\section{BIBLIOGRAPHIE}

\section{Sources primaires}

ARCHIVES NATIONALES

F 23/2. - Réfugiés, recherches de familles.

F 23/3. - Réfugiés : circulaires et instructions (1914-1919), libérés et rapatriés (1917-1919).

F 23/4. - Journaux de réfugiés.

F 23/12. - Rapatriement, généralités.

F 23/15. - Rapatriement d'enfants français.

Série AJ 4. - Interrogatoires de rapatriés dans leur département d'accueil.

SERVICE HISTORIQUE DE LA DÉFENSE

$5 \mathrm{~N}$ 367. - Rapatriés (coupures de presse).

5 N 377. - L'Ardennais de Paris.

$6 \mathrm{~N} 81$. - Rapatriés (fonds Clemenceau).

7 N 143. - Rapatriés (circulaire sur les rapatriés).

7 N 550-551. - Rapatriés (statistiques).

16 N 669. - Interrogatoires des réfugiés évacués par les Allemands, notes sur les habitants des pays envahis.

16 N 1226-1227. - Interrogatoires de rapatriés (1917-1918).

$16 \mathrm{~N}$ 1583. - Rapatriés (correspondance, centres de rapatriement).

17 N 441-442. - Évacuations des populations du Nord et du Pas-de-Calais.

18 N 191-192. - Interrogatoires des rapatriés.

19 N 355-356. - Renseignements fournis par les rapatriés sur l'organisation défensive allemande. 19 N 360. - Renseignements recueillis par la sûreté auprès des civils rapatriés.

ARCHives déPARTEMENTALES Des ARdenNes : Per H 27.

ARCHIVES DÉPARTEMENTALES DE LA MEUSE : 10 R 193.

\section{Sources secondaires}

ALIX Andrée D’ (1919), Le rapatriement : étude sur le rapatriement et ses œuvres de secours, Paris : Bloud \& Gay.

AMARA Michaël (2008), Des Belges à l'épreuve de l'Exil. Les réfugiés de la Première Guerre mondiale. France, Grande-Bretagne, Pays-Bas, Bruxelles : Éditions de l'Université de Bruxelles.

BECKER Annette (2012), Oubliés de la Grande Guerre. Humanitaire et culture de guerre, Paris : Pluriel.

ChAPTAL De CHANTEloup Léonie (1919), Les rapatriés 1915-1918, Paris : Librairie Alcan.

DERUELLE Eugénie (2010), Les carnets d'Eugénie Deruelle : une civile en zone occupée, Amiens : Encrage.

GATRELl Peter \& ZhVANKo Liubov (2017), Europe on the Move: Refugees in the Era of the Great War, Manchester : Manchester University Press.

HoRNE John \& KRAMER Alan (2001), German Atrocities, 1914: A History of Denial, New Haven et Londres : Yale University Press [trad. française 2005, 1914. Les Atrocités allemandes, Tallandier]. 
HoRne John \& Kramer Alan (1994), « German “Atrocities” and Franco-German Opinion, 1914: The Evidence of German Soldier's Diary », Journal of Contemporary History, 66(1), 1-33.

HUBER Michel (1931), La guerre et la vie sociale : la population de France pendant la guerre, Paris : PUF. KRAMER Alan (1994), « Les “Atrocités allemandes” : mythologie populaire, propagande et manipulation dans l'armée allemande », J. J. Becker, Guerre et culture, 1914-1918, Paris : Armand Colin, 147-164.

LAMBERT Jacques (1995/1996), « Louise et Fritz : un amour né de la Grande Guerre », Terres ardennaises, 52 (octobre 1995), 29-42 et 54 (mars 1996), 44-52.

LE NAOUR Jean-Yves (2000), «Femmes tondues et répression des “femmes à Boches” en 1918 », Revue d'histoire moderne et contemporaine, 47(1), 148-158.

McPhaIL Helen (2001), The Long Silence: Civilian Life under the German Occupation of Northern France, 1914-1918, Londres et New York : I. B. Taurus.

Nivet Philippe (2004), Les réfugiés français de la Grande Guerre, 1914-1920 : les « Boches du Nord », Paris : Economica.

NiVET Philippe (2011), La France occupée, 1914-1918, Paris : Armand Colin.

POURCHER Yves (1995), Les jours de guerre : la vie des Français au jour le jour entre 1914 et 1918, Paris : Hachette-Pluriel.

Proctor Tommy (2010), Civilians in a World at War, 1914-1918, New York : NYU Press.

RICHARD Ronan (1998), « Réfugiés, prisonniers et sentiment national en milieu rural en 1914-1918 : vers une nouvelle approche de l'Union sacrée ", Annales de Bretagne et des pays de l'Ouest, 105(4), 111-128.

RICHARD Ronan (2002), " “Étrangers" et "indésirables" en temps de guerre ", Annales de Bretagne et des pays de l'Ouest, 109(4), <http://journals.openedition.org/abpo/1523> (19 mars 2018).

STEPHANI Philippe (1919), Sedan sous domination allemande, 1914-1918, Paris : Grasset.

STIBBE Matthew (2006), « The Internement of Civilians by Belligerent States during the First World War and the Response of the International Commitee of the Red Cross ", Journal of the Contemporary History, 41, 5-19.

\section{ANNEXES}


Annexe 1. - Photographie de rapatriées à leur arrivée à Évian en 1917.

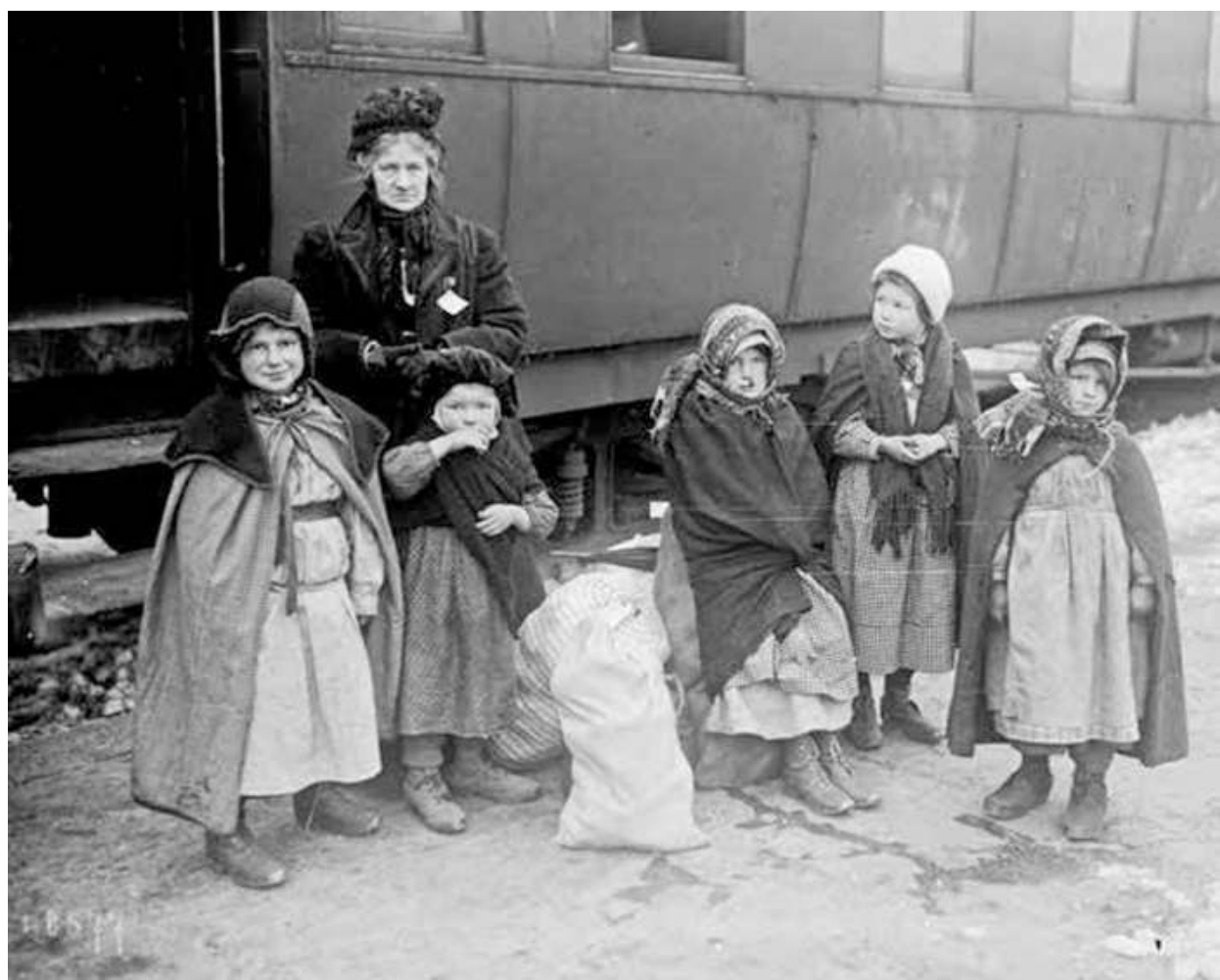

Collection Nicolas Charles.

Annexe 2. - Photographie de rapatriées à leur arrivée à Évian en 1917.

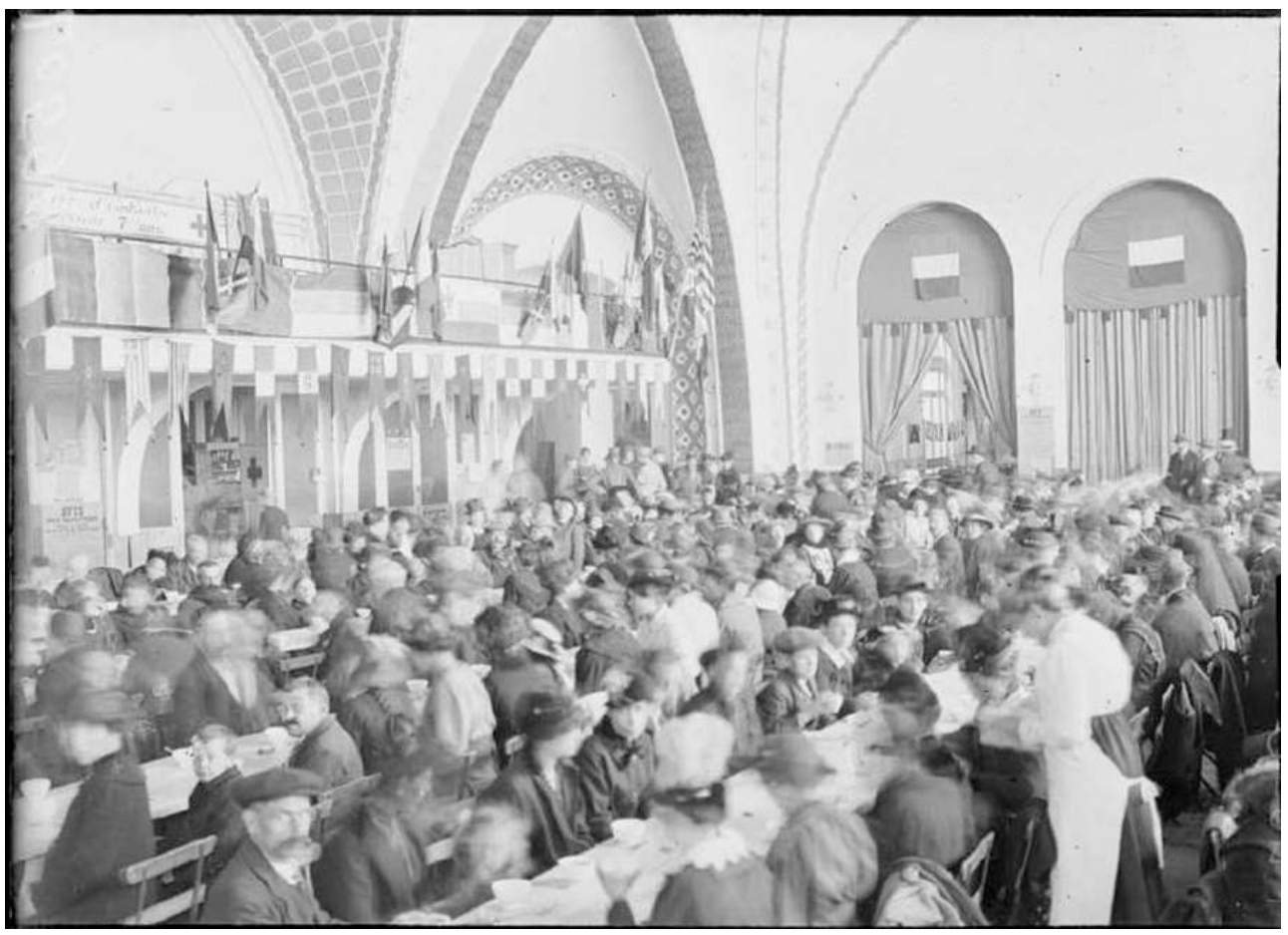

Collection Nicolas Charles. 


\section{NOTES}

1. Viols, meurtres de civils, mais aussi mains coupées et autres mutilations. Nous y reviendrons plus loin. Voir Horne \& Kramer (2001) et Kramer (1994).

2. Comme le maire de Charleville (Ardennes) en 1914, Edmond Bouchez-Leheutre, qui fait partie de la bourgeoisie locale.

3. Les déplacés, que l'on peut aussi qualifier d'évacués, sont les personnes qui ont dû quitter leur domicile à cause d'une armée. Celle-ci les a obligés à quitter leur domicile à cause du déroulement des combats. À partir de 1915, les Allemands vont proposer des évacuations de civils (surtout des «bouches inutiles» comme des femmes âgées ou des enfants dans un premier temps) afin de mieux gérer le manque de nourriture croissant dans les territoires occupés.

Sur les réfugiés durant la Première Guerre mondiale et leur statut, voir les travaux de P. Nivet (2004). Les réfugiés sont ceux qui fuient de leur propre gré mais dont l'État légitime la fuite, les évacués sont ceux que les autorités forcent à fuir. Ces deux catégories renvoient aux exodes d'août 1914 et du printemps 1918 et le ministère de l'Intérieur valide leur statut en publiant des listes de communes dont les originaires deviennent des ayants droit de l'assistance d'État (allocation aux réfugiés notamment).

4. Les deux gros flots de réfugiés de la Première Guerre mondiale sont en août-septembre 1914 puis au printemps 1918.

5. À partir de 1916-1917, les effets du blocus maritime allié se faisant sentir sur les Empires centraux, les Allemands décident d'évacuer un grand nombre de personnes : entre 1915 et 1918, plus de 500000 habitants des régions occupées sont évacués vers la France via la Suisse. Parmi eux, une majorité de femmes.

6. Ce n'est pas le cas des réfugiées et évacuées qui n'ont généralement pas connu l'ennemi, elles ont fui avant son arrivée. Toutefois, les populations de l'arrière ne savent pas souvent si les migrants ont connu l'ennemi, la suspicion est surtout liée à des paramètres sociaux, économiques et culturels, nous le verrons plus loin.

7. L'incompréhension est aussi linguistique : durant la Grande Guerre, malgré les progrès de la langue française dus au développement de l'instruction primaire depuis les lois Ferry de 1881-1882, les populations continuent de s'exprimer entre elles dans les dialectes locaux. Ainsi, les femmes qui arrivent dans le Midi se trouvent face à des personnes qui communiquent entre elles dans un dialecte occitan. Elles-mêmes utilisant leur propre dialecte, l'incompréhension linguistique est parfois totale.

8. Voir sur cet aspect Albert Bertier de Sauvigny, Pages d'histoire locale 1914-1919. Notes journalières et souvenirs, réédité en 1994 par l'association Soissonnais 14-18.

9. Les deux grandes vagues de réfugiés durant la Première Guerre mondiale (été 1914 et printemps 1918) ont provoqué le déplacement de près de 1,5 million de personnes.

10. Voir Horne \& Kramer (1994).

11. Service historique de la Défense, situé au château de Vincennes près de Paris, qui regroupe les archives de l'armée de terre française de la Grande Guerre.

12. C'est le cas du procès de Leipzig en 1921 où des soldats et officiers allemands sont jugés pour crimes de guerre. La tenue de ce procès était une des clauses du traité de Versailles du 28 juin 1919.

13. Bataille du 15 au 23 août 1914 où 674 civils ont été exécutés par les Allemands en représailles. 14. Cette remarque est encore plus vraie pour l'invasion de 1940 : l'énorme exode déclenché en mai 1940 par l'avancée des troupes d'Hitler est en grande partie dû aux invasions précédentes ; les civils du nord-est de la France ne voulant alors pas revivre une troisième occupation en soixante-dix ans se jettent massivement sur les routes pour fuir vers le sud. 
15. Il s'agit ici de l'armée française qui a évacué, lors de la stabilisation du front à l'automne 1914, de nombreux civils français dont le domicile se situait à proximité immédiate des combats.

16. Lettre du général Graziani, sous-chef d'état-major de l'armée, 17 mai 1915, Archives nationales, $7 \mathrm{~N} 143$.

17. De la gare de Genève à Annemasse, un tramway transporte les évacués.

18. Rapport du commissaire spécial d'Annemasse sur l'évacuation de la population, 31 mars 1915, Archives départementales de Haute-Savoie, 4 M 342.

19. Ibid., 17 avril 1915.

20. Ibid., 22 mars 1915.

21. Ibid., 31 mars 1915.

22. Voir le témoignage d'Alcide Aubert étudié par Marie-France Barbe, dans «La guerre en pays envahi, cahier d'Alcide Aubert ", Au pays des Rièzes et des Sarts. Annales d'histoire locale, $\mathrm{n}^{\text {os }} 83$ et 84 , 1980.

23. Rapport du commissaire spécial d'Annemasse sur l'évacuation de la population, 27 décembre 1915, Archives départementales de Haute-Savoie, 4 M 342.

24. «La rosserie et le locataire [l'Allemand qu'elle loge et sa maîtresse] seraient trop heureux d'avoir à eux toute ma maison ; et puisque j'ai souffert dix-sept mois, je continuerais jusqu'à la fin. » (12 mars 1916)

25. Rapport du commissaire spécial d'Annemasse sur l'évacuation de la population, 14 juin 1917, Archives départementales de Haute-Savoie, 4 M 342.

26. Ibid., 17 décembre 1916.

27. Notice individuelle de Louise Thieffry, 5 juin 1917, Archives départementales de HauteSavoie, 5 M 517.

28. Régulièrement les Allemands envoyaient des civils vers l'Allemagne dans des camps comme otages pour obliger les autres personnes de la commune à effectuer les travaux forcés ou les réquisitions.

29. Lille avait 217000 habitants avant la guerre, il n'en reste plus que 112000 à la libération de la ville en octobre 1918.

30. Rethel possède 5187 habitants au recensement de 1911.

31. Pour ces chiffres sur les Ardennes, voir Les Ardennes durant la Grande Guerre, CharlevilleMézières, 1994 (ouvrage collectif).

32. Cet exode reste le plus massif et le plus marquant dans l'imaginaire collectif français du $x^{e}$ siècle : tous les départements de la frontière nord-est de la France ont été en mai 1940 quasiment vidés de leur population, ce qui n'est pas le cas durant le premier conflit mondial.

33. Ici, depuis Avaux-le-Château dans le sud des Ardennes, près de la ligne de front, le jeune rapatrié est ensuite allé à Laon où il a été mis dans un convoi ferroviaire qui est passé par Hirson, Mézières puis est rentré en territoire allemand à Metz, Strasbourg. Ensuite, destination la Suisse via Zurich puis arrivée en France à Annemasse.

34. C'est-à-dire, pour les autorités françaises, les personnes qui ne collaborent pas avec les Allemands.

35. Celles qui sont signalées comme collaborant avec les Allemands ou tout du moins dont la vie quotidienne est facilitée par ces derniers.

36. Son signalement est ensuite fourni par le rapatrié interrogé. Cela est pris au sérieux par le fonctionnaire qui demande en note à la fin du procès-verbal : «Une enquête sera faite tant à Reims qu'à Épernay où se trouve le frère du dénommé T., à l'effet d'avoir tous les renseignements utiles sur les deux, notamment au point de vue national. »

37. C'est le cas par contre en Belgique où de nombreux cas de femmes tondues sont avérés en 1918. Voir Benoît Majerus, "Sex in the city : la prostitution à Bruxelles pendant la Grande Guerre (1914-1918)», Les cahiers de la Fonderie, n³2, 2005, p. 51-54. Voir aussi Le Naour (2000 : 148-158). 
38. C'est le cas en France à la Libération en 1944 : la vacance du pouvoir avant l'installation de l'administration du GPRF est à l'origine, pendant quelques jours, et ce sur tout le territoire, de cas de pendaisons sommaires de personnes suspectées d'avoir collaboré ou de femmes tondues pour avoir eu des relations intimes avec des Allemands.

39. Voir en annexe les photographies de rapatriées à leur arrivée à Évian en 1917.

40. Ceci pour éviter que d'éventuels espions ne se retrouvent à l'arrière des lignes françaises et renseignent les puissances centrales sur les dispositifs militaires alliés.

41. En France, depuis les lois scolaires de 1881-1882, l'obligation scolaire pour les filles et les garçons va de 6 à 13 ans.

42. Le dossier contenant les copies des enfants meusiens émigrés dans les Alpes-Maritimes date de 1916.

43. Dès 1929, Michel Huber les évaluait entre 1,8 et 2,3 millions tout en reconnaissant que l'ensemble des migrants, assistés et non assistés, avait pu atteindre les 3 millions (Huber, 1931).

44. Médaille des victimes de l'Invasion, surtout remise pour des actes de résistance ou de déportation; médaille de la Reconnaissance française, donnée aux civils qui ont servi le pays, surtout dans le cadre d'actes de résistance ou pour des missions médicales.

\section{RÉSUMÉS}

Cet article traite des femmes françaises (réfugiées, évacuées ou rapatriées), obligées de quitter les régions du Nord et du Nord-Est occupées durant la Grande Guerre. Qui sont celles qui quittent durant tout le conflit les dix départements passés sous domination ennemie? Dès les premiers jours de l'offensive allemande en 1914, beaucoup de Français menacés par l'avancée germanique quittent leur domicile pour aller vers Paris ou l'ouest du pays. Passée l'invasion, avec la stabilisation du front après novembre 1914, les réfugiés continuent quand même à affluer vers la «France libre », évacués par les Allemands qui souhaitent se débarrasser des " bouches inutiles ». Comment ces femmes sont-elles accueillies en France où elles sont, aux yeux de beaucoup, suspectes de collaborer, parfois de façon intime, avec l'ennemi ? Quels sont les dispositifs mis en place par l'État français pour les interroger, les recevoir, les loger?

Notre article sur ces réfugiées françaises durant la Première Guerre mondiale sera organisé en trois parties. Tout d'abord, il s'agira de dresser une typologie de ces femmes réfugiées. Pourquoi partent-elles? Qui sont celles qui partent? Ce seront les deux axes essentiels de cette partie. Ensuite, nous chercherons à voir comment l'État français s'occupe des réfugiées et des rapatriées françaises à leur arrivée en provenance des territoires occupés. Des logements sont fournis, et des interrogatoires pratiqués par des commissariats spéciaux à leur entrée sur le territoire via la Suisse (souvent en Haute-Savoie ou dans le Doubs). Ensuite, elles sont envoyées sur tout le territoire où les interrogatoires se poursuivent. Enfin, nous examinerons l'image qu'ont ces réfugiées auprès des autres français : nous verrons que l'image ambiguë de "Boche du Nord » leur a été attribuée, car la plupart des Français, loin du théâtre des opérations, avaient du mal à comprendre comment des Françaises avaient pu vivre au contact de l'ennemi. De par leur origine, ces femmes sont rapidement suspectées par l'opinion publique d'être des «femmes à Boches ", ce qui, dans le contexte de la guerre en fait de mauvaises françaises. Nous tâcherons donc ici de voir aussi comment ces femmes émigrées ont vécu cette situation, loin de chez elles, victimes de la guerre mais suspectées par leurs compatriotes. 
This article deals with the women who were forced to-or chose to-leave the Northern and Northeastern regions of France under German occupation during the Great War. Who were those who left, from 1914 to 1918, the ten departments that had passed under enemy domination? From the first days of the German offensive in 1914, many French people threatened by the German advance left their homes to go to Paris or the Western part of France. Following the invasion, and the stabilization of the front line after November 1914, refugees continued to flock to non-occupied zones-some of them evacuated by the Germans who wanted to get rid of "useless mouths". How were these women welcomed in these parts of France where many suspected them of having occasionally intimately collaborated with the enemy? What were the devices implemented by the French state to interrogate them, receive them, and accommodate them?

Our article will be organized in three parts. We will first draw a typology of these women. Why did they leave? Who were those who left? We will next look at how the French state dealt with French refugees arriving from the occupied territories. Housing was provided and interrogations led by special police forces when these entered the territory through Switzerland (often in Haute-Savoie or in the Doubs), before the refugees were sent throughout the country where interrogations continued. Finally, we will examine how these refugees were perceived by the rest of the population: we will see that they were given the ambiguous name of "Boches from the North" as most of the French population, who lived far from the occupied zones, found it hard to understand how French women might have lived in contact with the enemy. These women, because of their origin, were thus rapidly suspected by public opinion of having slept with the enemy and of being, therefore, unpatriotic. We will also try to see how these emigrants experienced this situation, far from home, victims of the war but suspected by their compatriots.

\section{INDEX}

Mots-clés : Première Guerre mondiale, femmes, « Boches du Nord », réfugiées, rapatriées, évacuées

Keywords : World War One, women, refugees, repatriated, "Boches from the North"

\section{AUTEUR}

\section{NICOLAS CHARLES}

Nicolas Charles est professeur agrégé, doctorant à Paris 1 Panthéon-Sorbonne sous la direction de Nicolas Offenstatdt et Alya Aglan sur le thème des Relations interpersonnelles entre civils français et soldats allemands dans le Nord et les Ardennes occupés entre 1914 et 1918. Il fait partie de l'UMR SIRICE. Membre de l'APHG, auteur de nombreux articles pour la revue Historiens \& Géographes, notamment dans l'« Abécédaire de la Grande Guerre » paru dans la revue en 2015. Il fait aussi des recensions d'ouvrages d'histoire contemporaine pour le site <Nonfiction.fr>. Il a rédigé avec Yohann Chanoir : Enseigner 14-18 : les mémoires de la Grande Guerre (éditions Canopé, 2015). Il a collaboré à plusieurs ouvrages collectifs sur la Grande Guerre ou sur l'histoire francoallemande dont le dernier en date, sous la direction de Rainer Bendick, Ulrich Bongertmann, Marc Charbonnier, Martin Stupperich et Hubert Tison : Deutschland und Frankreich: Geschichtsunterricht für Europa. Die deutsch-französischen Schulbuchgespräche. Erfolge - Perspektiven Desiderate. / France - Allemagne : l'enseignement de l'histoire pour l'Europe. Les rencontres francoallemandes sur les manuels scolaires. Succès - Perspectives - Desiderata (Wochenschau Verlag, 
Francfort-sur-le-Main, 2018).

nicolas.charles2@wanadoo.fr 